\title{
10 Jahre Arbeitsgruppe Geriatrische Onkologie der DGHO/DGG/AIO
}

\author{
Carsten Bokemeyer, für die interdisziplinäre Arbeitsgruppe Geriatrische Onkologie \\ II. Medizinische Klinik und Poliklinik, Onkologie, Hämatologie und Knochenmarktransplantation mit Sektion Pneumologie, \\ Hubertus-Wald-Tumorzentrum (UCCH), Universitätsklinikum Hamburg-Eppendorf, Deutschland
}

\begin{abstract}
Die Arbeitsgruppe Geriatrische Onkologie wurde anlässlich der Jahrestagung der Deutschen Gesellschaft für Hämatologie und Onkologie 1999 in Jena ins Leben gerufen. Der Anlass dazu war, dass trotz der enormen Bedeutung von Krebserkrankungen bei älteren Menschen hierzu wenig wissenschaftliche Erkenntnisse vorlagen und das Problemfeld geriatrische Onkologie noch keine wesentliche Beachtung erfuhr. Die Arbeitsgruppe ist mittlerweile interdisziplinär in der Deutschen Gesellschaft für Hämatologie und Onkologie (DGHO), der Deutschen Gesellschaft für Geriatrie (DGG) und der Arbeitsgemeinschaft Internistische Onkologie (AIO) der Deutschen Krebsgesellschaft verankert. Während konsequenterweise in den ersten Jahren der Tätigkeit der Arbeitsgruppe der Schwerpunkt in der Sensibilisierung der medizinischen Fachwelt für das Themengebiet der «geriatrischen Onkologie» lag, so hat sie in den nachfolgenden Jahren wichtige Beiträge zur Analyse der Therapieergebnisse bei der Behandlung onkologischer Erkrankungen älterer Patienten leisten können. In zahlreichen Übersichtsarbeiten und Literaturanalysen, die v. a. in ONKOLOGIE publiziert wurden, ist der anfangs noch begrenzte, jedoch stetig wachsende Umfang der Erkenntnisse in der Behandlung einzelner Tumorentitäten bei älteren Menschen dargestellt worden. Der zweite Schwerpunkt der Arbeitsgruppe lag in der Erarbeitung von Assessmentinstrumenten für die Praxis: Schon früh reifte die Erkenntnis, dass die gängigen Kriterien Performancestatus und «Blick des Arztes» bei älteren Patienten nicht ausreichen, um alle Faktoren zu erfassen, die vor Einleitung einer krebsspezifischen Therapie in dieser Altersgruppe notwendig sind. Die Einführung geriatrischer Assessmentinstrumente in die Onkologie für Therapieentscheidungen und die Überprüfung des prognostischen Wertes dieser Tests war daher die zentrale Aufgabe der interdisziplinären Arbeitsgruppe in den letzten Jahren. Zahlreiche der entwickelten Testinstrumente wurden von deutschen Studiengruppen auch im Rahmen ihrer multizentrischen Studien, so zum Beispiel bei den Non-Hodgkin-Lymphomen und der chronischen lymphatischen Leukämie, beim Mammakarzinom und beim Ovarialkarzinom, aufgegriffen. Damit soll eine bessere Beurteilung von Prognose des Patienten, Therapierbarkeit und potenzieller Therapienebenwirkung erarbeitet werden. Die ersten Ergebnisse dieser wichtigen Arbeiten werden für die Fachwelt in den nächsten Jahren zur Verfügung stehen, sodass dann zunehmend auch spezifische Studiendaten über die Therapie älterer onkologischer Patienten vorliegen dürften. Das ist die Grundlage für evidenzbasierte Empfehlungen für Behandlungskonzepte in dieser Altersgruppe.
\end{abstract}

Die weiteren Aktivitäten der Arbeitsgruppe während der letzten zwei Jahre betreffen die Versorgungsrealität in Deutschland. Mit Unterstützung der Initiative Geriatrische Hämatologie und Onkologie (IN-GHO) und eines Educational Grant der Firma Ortho Biotech wurde ein deutschlandweites Register zur Versorgungsrealität älterer Tumorpatienten geschaffen. Das Ziel ist dabei, die Einschätzung älterer Tumorpatienten durch ein strukturiertes, kurz gefasstes geriatrisches Assessment gegenüber der «gefühlten» Einschätzung des Arztes außerhalb von Studien zu validieren und die realen Therapieergebnisse mit diesen Einschätzungen zu überprüfen. Die Beobachtungszeit pro Patient umfasst ein halbes Jahr, und mittlerweile sind die Daten von über 2000 Patienten im Register verfügbar. Der Abschluss der Gesamtrekrutierung mit $3000 \mathrm{~Pa}-$ tienten ist für 2010 geplant. Bereits jetzt ergeben sich aus den ersten Pilotprojekten wichtige Erkenntnisse, die auf internationalen Kongressen, so z. B. dem Kongress der American Society of Hematology im letzten Winter und der American Society of Clinical Oncology in diesem Jahr in Orlando, präsentiert wurden. Auch die DGHO-Tagung 2009 hat auf das Thema geriatrische Onkologie hingewiesen - mit dem Motto «Gesellschaft der Methusaleme». Dieses Thema, zusammenfallend mit dem 10-jährigen Jubiläum der interdisziplinären Arbeitsgruppe der DGHO/DGG/AIO, war Anlass für die Erstellung dieses Sonderheftes. Langjährige Mitarbeiter der Arbeitsgruppe fassen in dieser Ausgabe wichtige Ergebnisse der letzten Jahre zusammen, so zu Aspekten der Versorgungsrealität, Register und Studienaktivitäten in der geriatrischen Onkologie, zum geriatrischen Assessment, zu Besonderheiten der Therapie bei älteren Tumorpatienten sowie zu Lebensqualität und Ethik. Dies alles wird in seiner Bedeutung vor dem Hintergrund des demografischen Wandels, der im einleitenden Beitrag als große politische Herausforderung dargestellt wird, noch wichtiger werden. Abgerundet wird das Sonderheft durch eine Betrachtung der biologischen Grundlagen von Krebs und Alterungsprozessen. Mit ihrem 10-jährigen Jubiläum ist die Arbeitsgruppe Geriatrische Onkologie immer noch jung. Die Herausforderungen werden weiter wachsen, so dass vielfältige Unterstützung auf individueller, sachbezogener und politischer Ebene für dieses Thema unerlässlich ist. Den Autoren und langjährigen Mitarbeitern der interdisziplinären Arbeitsgruppe möchte ich für ihren unermüdlichen Einsatz für unser Thema und für ihre informativen, interessanten Beiträge in diesem Heft danken. Ich wünsche den Lesern eine interessante Lektüre, die zum Nachdenken anregt.

Hamburg, im September 2009

Prof. Dr. Carsten Bokemeyer

\section{KARGER \\ Fax +497614520714 (c) 2009 S. Karger GmbH, Freiburg \\ Accessible online at: \\ E-mail Information@Karger.de www.karger.com/onk \\ www.karger.com}

Prof. Dr. med. Carsten Bokemeyer

II. Medizinische Klinik und Poliklinik, Onkologie, Hämatologie

und Knochenmarktransplantation mit Sektion Pneumologie

Universitätsklinikum Hamburg-Eppendorf

Martinistraße 52, 20246 Hamburg, Deutschland

Tel. +49 40 42803-2960, Fax -8054, c.bokemeyer@uke.uni-hamburg.de 